\title{
Cédric VILLANI, Les Mathématiques sont la poésie des
} sciences

\section{Mehdi K. Benslimane}

\section{(2) OpenEdition}

1 Journals

\section{Édition électronique}

URL : https://journals.openedition.org/ress/4699

DOI : $10.4000 /$ ress.4699

ISBN : $1663-4446$

ISSN : $1663-4446$

Éditeur

Librairie Droz

Édition imprimée

Date de publication : 30 mai 2019

Pagination : 295-299

ISSN : 0048-8046

Référence électronique

Mehdi K. Benslimane, «Cédric VILLANI, Les Mathématiques sont la poésie des sciences 》, Revue européenne des sciences sociales [En ligne], 57-1 | 2019, mis en ligne le 23 janvier 2019, consulté le 04 janvier 2023. URL : http://journals.openedition.org/ress/4699; DOI : https://doi.org/10.4000/ress. 4699

Ce document a été généré automatiquement le 4 janvier 2023.

Tous droits réservés 


\title{
Cédric VILLANI, Les Mathématiques sont la poésie des sciences
}

\author{
Mehdi K. Benslimane
}

\section{RÉFÉRENCE}

Cédric VILLANI, 2018 (2015), Les Mathématiques sont la poésie des sciences, Paris, Flammarion « Champs sciences », 128 p.

Les Mathématiques sont la poésie des sciences. Une phrase séduisante, poétique même. C'est le titre choisi qui explique vraisemblablement, après coup, l'attrait exercé par ce petit livre comme du reste par la science ainsi que par la poésie. La phrase en question n'est pas l'œuvre d'un mathématicien mais bien entendu d'un... poète, Léopold Sédar Senghor en l'occurrence. L'anecdote, citée au début du livre, est intéressante à raconter. Lors d'une réunion entre Hamet Seydi, mathématicien sénégalais, ancien doyen de la Faculté des sciences et techniques de l'université Cheikh Anta Diop de Dakar, et Senghor, président de la République du Sénégal d'alors, ce dernier qui devait prononcer l'allocution d'ouverture d'un congrès international de mathématiciens semble attiré par les titres choisis pour les différentes interventions programmées, avant de lancer à son interlocuteur: «Vous, les mathématiciens, vous faites de la poésie des sciences!». L'auteur du livre, Cédric Villani, est un brillant mathématicien, titulaire de la médaille Fields en 2010 (le «Nobel» des mathématiques) et élu à l'Académie des Sciences en 2013. Villani précise, d'entrée de jeu, qu'il opte pour la mathématique au singulier et non pour les mathématiques puisqu'il a compris depuis longtemps qu'« il ne pouvait donner aucune justification au pluriel » (p. 9).

2 Dans « Science es-tu là », son premier chapitre, il insiste pour dire que la mathématique est bel et bien une science, et en fait la démonstration. Scepticisme a priori, usage du raisonnement, partage des preuves, validation par les pairs (p.11); voilà ce qui en fait une science. Villani ne démontre pas seulement par des exemples, des chiffres et des preuves le caractère hautement scientifique de la mathématique, il défend la 
" supériorité » en quelque sorte de sa discipline sur les autres sciences. Ainsi, il affirme par exemple : «L'hypothèse de Riemman est LA plus célèbre de toutes [...]. Aucun autre domaine de connaissance n'est aussi intransigeant. » (p. 12-13). Bien entendu, l'auteur nuance ses propos en soulignant le l'écart entre la réalité pratique de l'exercice mathématique par les mathématiciens d'une part, et l'idéal scientifique d'autre part. Ceci dit, il défend que la conceptualisation, contrairement aux autres sciences, distingue fondamentalement la mathématique dans le sens où il n'y pas d'allers-retours entre la théorie et l'expérience pratique (p. 14). Par ailleurs, si la mathématique avait été un art, ça aurait été le design, puisque, comme en mathématique, "on retrouve la même dualité entre, d'une part, l'harmonie, l'abstraction, l'esthétique, et d'autre part, le devoir d'efficacité » (p.15). Et si elle avait choisi d'être un genre littéraire, ce serait plutôt la poésie, d'où le titre-intuition inspiré de Senghor. En mathématique, on promeut « le beau, l'original et l'utile» (p. 15).

3 Point de contrainte, point de créativité. Ainsi peut-on résumer le deuxième chapitre "Contraintes et créativité». "Pour moi, les contraintes sont indissociables de la créativité ; elles sont même l'un des sept ingrédients que j'aime présenter comme étant le cœur de la création des idées.» (p. 23). Et, selon l'auteur, "la grandeur [de la mathématique] est de parvenir, avec aussi peu d'ingrédients et tant de contraintes, à des énoncés si nombreux et si inventifs » (p. 23-24). Des contraintes à « l'inspiration », il n'y a qu'un pas que Villani franchit dans le troisième chapitre dans lequel il montre comment des concepts mathématiques peuvent inspirer des œuvres poétiques. Dans ce sens, il cite l'exemple de M. Escher « dont l'œuvre picturale a exploré tant de notions mathématiques» (p.31) comme celle notamment de la récursivité - laquelle est la propriété de s'appliquer à soi-même.

4 "Créer des liens» est un autre chapitre instructif sur les liens à l'intérieur de la discipline. Contre l'idée d'incompatibilité entre théories que de nombreux auteurs de sciences sociales défendent, Villani plaide pour ceci, s'agissant des mathématiques: "On découvre ainsi des relations entre des concepts développés par des personnalités différentes, dans des buts différents, au sein de théories différentes, souvent formulées autrement. » (p. 37, souligné par nous). « Dans son travail, le mathématicien est amené à établir des connexions à l'intérieur de la mathématique entre des choses qui sont sans rapport les unes avec les autres. » (p. 36). Et Villani de citer des mathématiciens plus ou moins illustres comme Poincaré, Riemman, Gromov, Serre, etc. « entrés dans la gloire en découvrant des correspondances inattendues» (p. 36). Pour ce qui est du chapitre "Un univers portatif », l'auteur développe l'idée selon laquelle « la recréation, qui est la base de la démarche mathématique [à l'aide d'équations et de formules], touche à la poésie dans sa nature profonde » (p.42) dans la mesure où l'étymologie du mot poésie signifie «création » dans la langue grecque. Villani dresse ensuite dans «La forme des mots » un parallèle entre mathématique et poésie remarquant que cette dernière est une question de mots, de langage. "La mathématique est un langage, et c'est même le langage par excellence des sciences exactes. » Elle devient aussi une sorte de langue universelle (p. 47). Non sans ironie, Villani ne manque pas de faire un clin d'œil aux sciences humaines dans une note de bas de page: «on a parfois voulu l'utiliser [la mathématique] comme langage des sciences humaines, mais cela s'est révélé bien moins convaincant... » (p. 47).

5 Dans «Visionnaires ", le septième chapitre, l'auteur poursuit « le parallèle entre la création du mathématicien et celle du poète évoquant la passion de la création, 
l'angoisse de la page blanche, l'état de flot créatif, le rôle de l'intuition, ou encore celui de l'émerveillement» (p.54). "Nul ne peut être mathématicien s'il n'a l'âme d'un poète » écrivait la mathématicienne Sophia Kowalevskaya. Villani disserte, enfin, sur son propre style rappelant, au passage, le fait que les mathématiciens possèdent des styles différents à la fois dans la façon d'aborder les problèmes, de les écrire et de les résoudre : «dans ma vie de mathématicien, j’ai été amené à synthétiser les influences de différents confrères, un peu comme un artiste qui s'inspire d'untel et puis d'un autre, et qui crée son propre style en bâtissant, en partie en réaction à ses prédécesseurs, mais toujours aussi en s'en inspirant; et j'ai vu mon propre style évoluer sans que je puisse y faire grand-chose» (p.56). Le chapitre "Poincaré et l'omnibus » présente quant lui, comme le titre l'indique, l'histoire de la créativité vue par le célèbre mathématicien à partir d'un exemple raconté plus en détail à la fin du livre. Poincaré «y décrit le rôle des mécanismes inconscients, des analogies, dans la description d'une situation mathématique, le lien entre des éléments distincts, le rôle du nouveau regard porté sur un objet, celui de l'inspiration extérieure ». "Comment vient l'inspiration? Pas de règle...» nous dit l'auteur. « Elle arrive souvent en un déclic, après de longs moments de travail acharné. » (p. 59). Et Villani de conclure : «Cherchez l'inspiration à l'extérieur comme le faisait si bien Poincaré.» (p. 61). L'avant-dernier chapitre « Ping-pong » renvoie à un sport passionnément pratiqué par Villani durant sa jeunesse. Le tennis de table lui sert d'analogie pour renvoyer aux « interactions dans le métier de scientifique, aux échanges entre collaborateurs au quotidien » comme lors de congrès et séminaires ainsi qu'aux trajectoires - à l'instar de celle d'une balle dans une partie de ping-pong - des théories et des théorèmes mathématiques. (p. 65-66)

6 Enfin, dans le dixième et avant-dernier chapitre intitulé « Ode à l'imperfection » - texte issu d'un exposé au Milanesiana, un festival à Milan portant sur le cinéma, la littérature et les sciences -, l'auteur reprend les thèmes majeurs du livre comme la beauté, les contraintes, la création, etc. Avec une idée-force en filigrane : L'erreur est humaine, elle est aussi scientifique. «L'erreur n'est pas un simple obstacle sur la route : c'est aussi un élément du cheminement » (p. 84) écrit Villani en donnant l'exemple de Poincaré, et comment ce dernier s'est lourdement trompé au début sur le "problème des trois corps » avant d'arriver, finalement, à une grande découverte. «L'imperfection nous est familière. Nous baignons dans l'imperfection, nous sommes les enfants de l'imperfection, nous lui devons tout [...]. L'imperfection, statutaire et salutaire, est notre force ; tous parfaits, nous serions condamnés. » Les imperfections sont multiples : dans les langues, dans les programmes informatiques comme dans les inventions technologiques et même dans la pensée humaine. «Oui, c'est bien de l'imperfection que naissent les grands progrès » conclut le mathématicien (p. 82-86).

7 L'Invention mathématique, cette "prose délicieuse ", dont l'expression est empruntée à Henri Poincaré (Science et Méthode, 1908), sert de titre à l'ultime chapitre du livre. Ce dernier y raconte des histoires personnelles et témoigne surtout des circonstances de ses expériences passées. Il y suggère notamment qu'un bon mathématicien, c'est à la fois une très bonne mémoire et une grande force d'attention. Si le mathématicien qu'est Poincaré admet qu'il n'a pas une bonne mémoire, il pense savoir pourquoi sa mémoire ne lui fait pas défaut lorsqu'il s'agit d'établir un raisonnement compliqué : "Une démonstration mathématique n'est pas une simple juxtaposition de syllogismes: ce sont des syllogismes placés dans un certain ordre, et l'ordre dans lequel ces éléments sont placés est beaucoup plus important que ne le sont ces éléments eux-mêmes. Si j'ai le sentiment, l'intuition, pour ainsi dire, de cet ordre, de façon à apercevoir d'un coup 
d'œil l'ensemble du raisonnement, je ne dois plus craindre d'oublier l'un des éléments ; chacun d'eux viendra se placer de lui-même dans le cadre qui lui est préparé, et sans que j'aie à faire aucun effort de mémoire.» (p. 94). Ceci dit, Poincaré réfléchit sur ce qu'est l'acte d'inventer, qui est le fait de discerner, de choisir. «Le véritable travail de l'inventeur consiste à choisir entre [des] combinaisons, de façon à éliminer celles qui sont inutiles ou plutôt à ne pas se donner la peine de les faire.» (p. 104). Quid de l'élément esthétique dans l'acte de création mathématique? Le célèbre mathématicien s'interroge et tente une esquisse de réponse : "Quels sont les êtres mathématiques auxquels nous attribuons ce caractère de beauté et d'élégance et qui sont susceptibles de développer en nous une sorte d'émotion esthétique ? Ce sont ceux dont les éléments sont harmonieusement disposés, de façon que l'esprit puisse sans effort en embrasser l'ensemble tout en pénétrant les détails.» (p. 107).

8 Faire de la science est-ce, dans un sens, faire de l'art? Si le scientifique, quelque soit sa discipline, crée des choses (des concepts, par exemple) - et il a besoin bien souvent d'indiscipline pour y arriver - il peut être considéré comme un artiste. Et c'est sans doute ceci qui le rend plus ou moins sensible au monde qui l'entoure. La réponse à cette question par la positive se comprend également si l'on garde à l'esprit le fait que le scientifique conçoit et met en place des "plans" un peu comme l'architecte; l'architecture étant considérée comme le premier art. Tout le long du livre, le mathématicien dresse des parallèles entre sa discipline et la poésie. Pour ce qui nous concerne, on peut se demander, à juste titre, à quel(s) art(s), voire à quels genres littéraires, s'apparenteraient le plus les nombreuses sciences humaines et sociales. Et chercher, par conséquent, les articulations de l'une à l'autre. L'histoire serait-elle proche du roman... historique ; une certaine science politique - étudiant les régimes politiques autoritaires ou non - du roman policier; l'anthropologie celle du récit de voyage, etc. ? En un mot, il s'agit d'un texte vif, intelligent, où l'on voit, au travers des lectures et des auteurs cités, l'amour que porte Villani à la poésie. Il demeure regrettable que l'auteur n'ait pas pu développer davantage son texte qui est, à l'origine, le support d'une conférence prononcée le 20 mars 2013 à la Maison de la culture de Namur, organisée conjointement par le département de mathématiques de l'université de cette ville et l'association « Les Midis de la poésie de Bruxelles ».

\section{AUTEURS}

MEHDI K. BENSLIMANE

IEP de Grenoble 